\title{
Frontières
}

\section{Marchandisation de l'hébergement des personnes âgées et enjeux éthiques}

\author{
Le point de vue des acteurs des réseaux public et privé
}

\section{Michèle Charpentier}

Volume 17, numéro 1, automne 2004

Au péril de l’accompagnement

URI : https://id.erudit.org/iderudit/1073605ar

DOI : https://doi.org/10.7202/1073605ar

Aller au sommaire du numéro

Éditeur(s)

Université du Québec à Montréal

ISSN

1180-3479 (imprimé)

1916-0976 (numérique)

Découvrir la revue

Citer cet article

Charpentier, M. (2004). Marchandisation de l'hébergement des personnes âgées et enjeux éthiques : le point de vue des acteurs des réseaux public et privé.

Frontières, 17(1), 42-47. https://doi.org/10.7202/1073605ar
Résumé de l'article

La privatisation des services de santé et des services sociaux soulève d'importants débats éthiques. Dans ce contexte, la très forte expansion des résidences privées pour personnes âgées suscite des inquiétudes quant au respect des droits des résidents : liberté individuelle, qualité de vie, accès aux soins et protection contre les abus. Se basant sur une étude qualitative et quantitative réalisée au Québec, cet article présente l'opinion des acteurs du réseau public et des propriétaires de résidences privées sur ces enjeux éthiques. Les résultats rendent compte de visions très différentes mais révèlent un consensus quant à la pertinence d'une intervention de l'État. Au-delà des préjugés, l'auteure souhaite alimenter le débat sur les impacts de cette marchandisation des services auprès des personnes âgées vulnérables et de ceux qui les accompagnent au quotidien. 


\section{Résumé}

La privatisation des services de santé et des services sociaux soulève d'importants débats éthiques. Dans ce contexte, la très forte expansion des résidences privées pour personnes âgées suscite des inquiétudes quant au respect des droits des résidents: liberté individuelle, qualité de vie, accès aux soins et protection contre les abus. Se basant sur une étude qualitative et quantitative réalisée au Québec, cet article présente l'opinion des acteurs du réseau public et des propriétaires de résidences privées sur ces enjeux éthiques. Les résultats rendent compte de visions très différentes mais révèlent un consensus quant à la pertinence d'une intervention de l'État. Au-delà des préjugés, l'auteure souhaite alimenter le débat sur les impacts de cette marchandisation des services auprès des personnes âgées vulnérables et de ceux qui les accompagnent au quotidien.

Mots clés: marchandisation - résidences privées pour personnes âgées - enjeux éthiques - droits des résidents.

\section{Abstract}

The privatisation of health and social services brings about important ethical debates. In this context, the phenomenal expansion of private residences for the elderly raises worried concerning the residents' rights (liberty of choice, quality of life, access to health care and protection against abuse). Relying on a qualitative and quantitative study done in the Eastern Townships of Quebec, the author presents the opinion of the workers from the public sector and the residences' owners concerning these issues. The results show significant differences of visions but a consensus about the necessary of a governmental intervention to insure the rights of vulnerable residents. Bringing up ethical stakes underlying an eventual regulation of the private residences, the author goes beyond preconceived ideas to enrich the debate around the question.

Key words: privatization - homes for the aged - ethical issues - residents rights.

\section{Marchandisation de l'hébergement des personnes âgées et enjeux éthiques Le point de vue des acteurs des réseaux public et privé}

\section{Michèle Charpentier, Ph.D., professeure, École de travail social, UQAM.}

Sujet d'actualité, s'il est un, la place du privé dans le domaine de la santé et des services sociaux suscite inquiétudes et controverses. Déjà présent dans notre univers sociosanitaire, le privé prend divers visages; que l'on pense aux agences privées de services à domicile, à la soustraitance privée en milieu hospitalier, etc. (Soderstrom, 1987; Conseil de la santé et du bien-être, 1997). Nous nous intéressons depuis plusieurs années au phénomène d'expansion des résidences privées pour personnes âgées, particulièrement aux enjeux sociaux, éthiques et politiques qui y sont associés. Les résidences pour personnes âgées constituent un cas type de ressources marchandes qui se sont développées dans le contexte de transformation du rôle de l'État et le courant «anti-institutionnalisation». Cette marchandisation des services d'hébergement suscite des questionnements particuliers car elle s'adresse à une population dite vulnérable, soit majoritairement des femmes du grand âge.

Le présent article se propose, à la lumière de nos travaux de recherche (Charpentier, 2000; Charpentier et al., 2002), de brosser un portrait des résidences privées pour personnes âgées au Québec pour ensuite discuter des principaux enjeux éthiques en présence, tels qu'ils sont perçus par les acteurs des réseaux public et privé concernés. Dans ce libre marché des résidences, qu'en est-il des conditions de vie et du respect des droits fondamentaux des aînés: liberté individuelle, qualité de vie, accès aux soins requis et protection contre les abus et la maltraitance? Qu'en pensent les intervenants qui accompagnent ces aînés souvent fragilisés par une perte d'autonomie: les gens du privé (propriétaires de résidences) et les gens du public (gestionnaires des services de soutien à domicile et d'hébergement, travailleurs sociaux impliqués dans l'orientation et la relocalisation des personnes âgées)? Au-delà des idées préconçues, voilà quelques questions que nous voulons soulever et souhaitons alimenter à l'aide de données empiriques.

\section{LE MARCHÉ DES RÉSIDENCES PRIVÉES POUR PERSONNES ÂGÉES AU QUÉBEC}

L'existence d'institutions pour héberger les personnes âgées, tels les asiles, les hospices, les centres d'accueil et d'hébergement, gérées par des entreprises privées ou des communautés religieuses au Québec n'est pas récente (Vaillancourt et al., 1987; 
Vaillancourt, 1997; Charpentier, 2002). Ce n'est toutefois qu'à partir des années 1980 que l'on assiste à l'émergence d'un véritable marché des résidences privées à but lucratif. Cet essor du secteur marchand s'explique notamment par l'augmentation de la population âgée, surtout celle de 75 ans et plus qui est davantage confrontée aux limites de l'habitat en solo, et par la rationalisation de l'offre de services publics, dont la diminution du taux d'institutionnalisation des personnes âgées et l'insuffisance des services de soutien à domicile (Conseil des aînés, 2000; Charpentier, 2002). Les résidences privées viennent en quelque sorte prendre le relais entre un maintien à domicile, avec trop peu ou pas de ressources, et un placement en institution publique dont l'accès se voit réduit aux personnes en situation de grande dépendance.

Il s'avère toutefois difficile de faire un portrait de ce secteur d'activité effervescent puisqu'il s'est développé en marge du réseau public, sans contrôle étatique ou avec ce que Brissette (1992) qualifie de «silence permissif de l'État». C'est d'ailleurs pour pallier à ce manque de données fiables que la Loi sur les services de santé et services sociaux (LSSS) a été amendée en 2002 pour obliger les agences régionales à tenir un registre des résidences pour personnes âgées sur leur territoire, définies comme suit:

Une résidence pour personnes âgées est un immeuble d'habitation collective où sont offerts, contre le paiement d'un loyer, des chambres ou des logements destinés à des personnes âgées et une gamme plus ou moins étendue de services, principalement reliés à la sécurité et à l'aide à la vie domestique ou à la vie sociale, à l'exception d'une installation maintenue par un établissement et d'un immeuble ou d'un local d'habitation où sont offerts les services d'une ressource intermédiaire ou d'une ressource de type familial.

(Art. 346.0.1)

La définition retenue couvre une grande variété de ressources résidentielles, allant de la résidence familiale, convertie pour accueillir trois ou quatre personnes âgées, au luxueux complexe urbain avec ascenseurs, infirmerie, piscine, sauna et salon de coiffure. Les données du registre révèlent cependant que la moitié des résidences recensées sont de très petite taille (1-9 unités); la taille moyenne des résidences privées au Québec étant établie à 29,8 unités (Gouvernement du Québec, 2004; Aubry, 2004). Bien qu'encore incomplet et à plusieurs égards imprécis, le registre a recensé à ce jour 2500 résidences privées, accueillant 80550 personnes âgées; 87\% des résidences ayant complété une déclara- tion sont à but lucratif. Selon ces données, le secteur marchand constitue l'acteur principal de l'hébergement des personnes âgées au Québec, accueillant 8\% de la population âgée du Québec, soit deux fois plus que les ressources publiques et agréées par le réseau de la santé: CHSLD et ressources intermédiaires (Charpentier, 2002; MSSS, 2004). Étonnamment, le registre fournit peu d'informations sur le profil des résidents (sexe, statut marital, niveau d'autonomie, etc.) sauf sur leur âge: 32,3\% des personnes âgées vivant en résidence ont 85 ans et plus. Voilà pourtant une question essentielle, car si les résidences privées s'adressent en principe à une clientèle dite autonome ou semiautonome, les études ont démontré qu'elles hébergent dans les faits des résidents de plus en plus âgés et en perte d'autonomie physique et souvent cognitive (Vézina et al., 1994; Bravo et al., 1998; Charpentier, 2002). Il s'avère que de nombreux résidents, majoritairement des femmes du quatrième âge, présentent plusieurs facteurs de vulnérabilité: au niveau de leur santé physique et cognitive, au niveau social (effritement $\mathrm{du}$ réseau social et familial) et au niveau économique (précarité financière, pauvreté).

Compte tenu des besoins accrus et de la «fragilité» de la clientèle du privé, il y a lieu de s'interroger sur les effets négatifs d'un système basé sur la libre entreprise et les rapports marchands. Plusieurs travaux ont mis en évidence la faiblesse et le flou du cadre d'opération des résidences privées au Québec et de leur régulation (Brissette, 1992; Commission des droits de la personne et des droits de la jeunesse (CDPDJ), 2001; Charpentier, 2002). En bref, disons que les règles actuelles régissent essentiellement le bâtiment et qu'il n'existe aucune norme relative aux conditions de vie et aux services offerts dans les résidences. Les résidences pour personnes âgées ne sont pas tenues de posséder un permis du ministère de la Santé et des Services sociaux et elles ne sont pas soumises à une accréditation régionale. Elles doivent respecter les règlements municipaux concernant les ressources de chambres et pension, selon la taille de l'établissement. Quant aux mesures de protection pour contrer les situations abusives ou les mauvais traitements, prévues dans diverses législations (Code civil, Charte des droits et libertés et LSSS), les données que nous avons recensées et nos analyses ont démontré que les modalités d'application, basées sur un système de plaintes ou de signalement, limitent grandement leur portée et leur efficacité (Charpentier, 2002). La problématique d'ensemble soulève ainsi des enjeux sociaux et éthiques importants en ce qui a trait aux conditions de vie et au respect des droits des personnes âgées, surtout pour celles qui sont plus vulnérables. Il faut reconnaître, en contrepartie, que les centres d'hébergement CHSLD, qui sont normés, encadrés et réglementés, ne constituent pas pour autant un modèle de qualité. Ces derniers ne répondraient qu'à environ $60 \%$ des besoins de leur clientèle. Le défi du grand âge et de la qualité de vie en milieu d'hébergement s'étend aussi à nos institutions publiques (CDPDJ, 2001; MSSS, 2004).

\section{LES ENJEUX ÉTHIQUES: LE POINT DE VUE DES ACTEURS DES RÉSEAUX PUBLIC ET PRIVÉ}

La démarche empruntée pour analyser les enjeux éthiques que soulève l'expansion du marché des résidences privées s'appuie donc sur nos résultats de recherche, principalement sur nos travaux de doctorat publiés récemment (Charpentier, 2002). Le contexte des résidences privées y est donc discuté dans une perspective éthique, en regard des valeurs socialement reconnues, soit le respect des droits fondamentaux des citoyens âgés: liberté individuelle, qualité de vie, accès aux soins requis et protection contre toutes formes d'abus (Delpérée, 1991; Boitte, 1995; Boisvert, 1997). C'est avec la conviction qu'il faut donner la parole aux acteurs directement impliqués que la collecte de données fut entreprise sur le terrain, en combinant des méthodes quantitatives (questionnaire postal) et qualitatives (groupes de discussion) ${ }^{1}$. L'étude s'est déroulée en Estrie, une région urbaine et rurale, qui, notamment par sa réalité biculturelle, est considérée comme un baromètre représentatif du Québec. La population à l'étude était composée d'une part des propriétaires de résidences privées, et d'autre part des différents intervenants du réseau public qui œuvrent auprès des résidences privées ou sont directement concernés par leur développement (gestionnaires et intervenants responsables du dossier des résidences privées à la Régie régionale et au soutien à domicile en CLSC, coordonnateurs des soins en CHSLD, travailleurs sociaux en CLSC et centre de jour, etc.). Le questionnaire postal a été conçu spécifiquement pour cette étude et il a été acheminé à tous les acteurs des réseaux public et privé inventoriés. Sur 213 personnes sollicitées, 139 ont retourné leur questionnaire, soit un taux de participation de 65\%. L'échantillon final, comprenant 137 sujets, se répartit en deux groupes de taille relativement semblable: 65 répondants pour le secteur public et 72 pour le privé. Les deux groupes de répondants désignés privé-public, dont nous comparerons les perceptions, présentent des profils similaires en ce qui a trait au sexe et à l'âge. Nous sommes toutefois confrontés à un manque de données descriptives sur les non-répondants, ce qui constitue une limite de l'étude. 
TABLEAU 1 - RESPECT DES DROITS FONDAMENTAUX DES RÉSIDENTS SELON LES ACTEURS DU SECTEUR PUBLIC ET DU SECTEUR PRIVÉ

DANS QUELLE MESURE CONSIDÉREZ-VOUS QUE LES PERSONNES ÂGÉES QUI VIVENT EN RÉSIDENCE PRIVÉE:

\begin{tabular}{|c|c|c|c|}
\hline & $\begin{array}{c}\text { Gestionnaires et } \\
\text { travailleurs sociaux } \\
\text { Secteur-PUBLIC } \\
(n=65)\end{array}$ & $\begin{array}{c}\text { Propriétaires } \\
\text { d'une résidence } \\
\text { Secteur-PRIVÉ } \\
(n=72)\end{array}$ & $\begin{array}{c}\text { Comparaison } \\
\text { des } 2 \text { groupes } \\
\text { (valeur } p \text { ) }\end{array}$ \\
\hline $\begin{array}{r}\text { très peu } \\
\text { peu } \\
\text { assez } \\
\text { beaucoup }\end{array}$ & $\begin{array}{c}(n=65) \\
3(4,6 \%) \\
30(46,2 \%) \\
30(46,2 \%) \\
2(3,1 \%)\end{array}$ & $\begin{array}{c}(n=71) \\
6(8,4 \%) \\
18(25,4 \%) \\
34(47,9 \%) \\
13(18,3 \%)\end{array}$ & 0,006 \\
\hline $\begin{array}{rr}\begin{array}{l}\text { Ont une bonne } \\
\text { qualité de vie? }\end{array} & \begin{array}{r}\text { très peu } \\
\text { peu } \\
\text { assez } \\
\text { beaucoup }\end{array}\end{array}$ & $\begin{array}{c}(n=64) \\
3(4,7 \%) \\
23(35,9 \%) \\
36(56,3 \%) \\
2(3,1 \%)\end{array}$ & $\begin{array}{c}(n=72) \\
2(2,8 \%) \\
8(11,1 \%) \\
42(58,3 \%) \\
20(27,8 \%)\end{array}$ & $<0,001$ \\
\hline $\begin{array}{lr}\text { Ont accès aux soins } \\
\text { requis par } & \text { très peu } \\
\text { leur condition? } & \text { peu } \\
& \text { assez } \\
& \text { beaucoup }\end{array}$ & $\begin{array}{c}(n=64) \\
5(7,8 \%) \\
28(43,8 \%) \\
29(45,3 \%) \\
2(3,1 \%)\end{array}$ & $\begin{array}{c}(n=72) \\
3(4,2 \%) \\
10(13,9 \%) \\
34(47,2 \%) \\
25(34,7 \%)\end{array}$ & $<0,001$ \\
\hline $\begin{array}{lr}\text { Subissent de l'abus } \\
\text { de la part de } & \text { très peu } \\
\text { la résidence? } & \text { peu } \\
& \text { assez } \\
& \text { beaucoup }\end{array}$ & $\begin{array}{c}(n=63) \\
18(28,6 \%) \\
32(50,8 \%) \\
13(20,6 \%) \\
0\end{array}$ & $\begin{array}{c}(n=69) \\
50(72,5 \%) \\
16(23,2 \%) \\
2(2,9 \%) \\
1(1,4 \%)\end{array}$ & $<0,001$ \\
\hline
\end{tabular}

Il ne fait pas de doute à la lecture du tableau 1, sur le respect des droits des personnes âgées vivant en résidences privées, que les propriétaires de résidences privées $(n=72)$ expriment une perception différente des acteurs du réseau public $(n=65)$, laquelle se veut plus positive. Ce constat vaut pour toutes les valeurs appréciées, à l'exception de la liberté de choix qui a été jugée de façon similaire par les deux catégories d'acteurs. Il ne faudrait pas conclure toutefois que les acteurs du secteur public portent un jugement négatif sur l'éthique dans le secteur privé d'hébergement. Pour chacun des droits, l'opinion dominante exprimée révèle que le niveau de respect est «assez» satisfaisant et ce, dans une proportion variant de $45,3 \%$ à $69,2 \%$. Soulignons que les positions sont plus mitigées en ce qui a trait à l'accès aux soins et à la qualité de vie. Près du tiers des répondants du secteur public ont attribué à ces droits une cote reflétant un faible niveau de respect («peu»). Quant aux abus envers les résidents, les données recueillies vont dans le sens de la documentation, suggérant une faible prévalence du problème. Là encore une différence très significative s'observe entre les deux types d'intervenants; le secteur public juge (dans une proportion de 50,8\%) que «peu» de résidents en sont victimes alors que $72,5 \%$ des propriétaires considèrent que cette forme de maltraitance est «très peu» présente. Ces divergences d'appréciation «privé-public» et ce préjugé favorable des propriétaires ont aussi été mis en évidence lors des groupes de discussion, avec cependant moins d'acuité. Les discours tenus lors des deux groupes de discussion, réunissant séparément huit intervenants du réseau public de la santé et des services sociaux et six propriétaires de résidences privées, sont plus nuancés. Ces données qualitatives, combinées aux commentaires écrits à la fin des questionnaires contribuent à enrichir l'analyse des enjeux éthiques en présence ainsi que notre compréhension de la dynamique d'ensemble.

\section{LA LIBERTÉ DE CHOISIR SON MILIEU DE VIE}

En ce qui a trait au principe du choix libre et éclairé de sa résidence, de la liberté contractuelle, les intervenants rencontrés ont exprimé d'importantes réserves. Les témoignages relatant les circonstances entourant la sélection de leur futur milieu de vie sont éloquents. Selon plusieurs interlo- cuteurs, ce sont les enfants, surtout les filles, qui choisissent la résidence. Il arrive que la personne âgée n'a même pas visité les lieux avant d'emménager. Comme mentionné par une travailleuse sociale dans ses commentaires libres à la fin du questionnaire postal, le choix est aussi fortement conditionné par la capacité de payer des résidents:

La majorité des personnes aînées n'ont pas les ressources financières suffisantes pour un hébergement dans le réseau privé. À moins de choisir une résidence qui offre moins de services. C'est la même chose lorsque les gens ont besoin de convalescence en postopération en raison des diminutions de la durée de séjour.

Il appert que les résidents défavorisés financièrement et en perte d'autonomie n'ont pas la capacité «avec leur petite pension» de s'offrir une résidence privée qui dispense des soins plus spécialisés. L'étude de Bravo et al. (1998), citée précédemment, permet d'ailleurs d'estimer que $52 \%$ des résidents en perte d'autonomie dans les résidences privées vivent du supplément de revenu garanti. De nombreux intervenants du réseau public se sont dits préoccupés des inégalités actuelles et en appellent au principe d'équité. Ces données invitent à considérer la vulnérabilité physique, cognitive et financière de nombreux résidents comme des facteurs limitant significativement leur liberté de choix et leur autonomie décisionnelle. Le groupe de travail du Conseil de la santé et du bien-être social a déjà énoncé des mises en garde qui vont dans ce sens: «un malade ou toute personne qui éprouve des problèmes d'adaptation sociale ou qui a un besoin de protection ne constitue pas un interlocuteur commercial valable» (1997, p. 1). Pourtant, malgré toutes ces limites à l'exercice d'un choix «libre et éclairé», condition sur laquelle repose la validité d'un contrat (dont la location d'un bail), le régime de la liberté contractuelle et la loi du marché règnent sans susciter de remise en question.

LA QUALITÉ DE VIE EN RÉSIDENCE PRIVÉE

Les groupes de discussion et les commentaires recueillis dans le questionnaire mettent en évidence que la qualité de vie en résidence est une préoccupation commune à tous les acteurs et qui se caractérise par d'importantes disparités. Les propriétaires se sont avérés très soucieux de la réputation de leur résidence; ce qui contribue à expliquer l'appréciation si positive qu'ils ont fourni dans l'enquête postale. À leur avis, la force du privé réside dans sa capacité à recréer un climat plus personnalisé. Sept commentaires libres émis dans l'enquête valorisent les dimensions humaines des petits milieux. À leur lecture, on se sent 
loin du discours traditionnel tenu par des entrepreneurs privés.

I think it is very important that they keep these small homes for the elderly, so they can have a more family atmosphere than in institutions. They have more ears in small homes because of the time that you can give them. They have more freedom to do what they enjoy as to living in institutions.

Je viens de placer une personne de 87 ans Alzheimer (en CHSLD). Je réalise que je lui accordais plus de soins et de surveillance que ces 2 semaines passées à l'hôpital. Car un petit milieu est toujours à proximité de nous. Des gens sont même décédés chez nous à leur demande «Dieu soit loué». L'expérience est lourde mais tellement enrichissante plus humaine plus familiale. C'est un dur métier mais une grande vocation.

Si les acteurs du réseau public consultés font une distinction entre les ressources privées qui sont structurées et celles qui naissent de façon spontanée ou quasi «improvisée», ils notent un manque flagrant de formation chez les propriétaires. Ils ne comprennent pas que n'importe qui puisse ainsi s'improviser et ouvrir une résidence sans un minimum de qualifications. Ce laxisme de la société au chapitre de la compétence des propriétaires et de leur personnel leur apparaît d'autant plus incompréhensible que leur clientèle est âgée, vulnérable et présente des pertes d'autonomie allant de légères à sévères.

Actuellement, je constate que les clientèles en résidence privée s'alourdissent, mais que les ressources humaines et matérielles n'augmentent pas. Les résidences privées sont au bord de la crise. Le gouvernement favorise le maintien des gens dans les résidences privées par «l'alternative à l'hébergement», mais il ne s'est pas assuré que ces résidences pourront répondre à ce contrat. (travailleur social)

\section{L'ACCÈS AUX SOINS REQUIS}

Il ressort des échanges de la rencontre de groupe réunissant des intervenants du réseau de la santé que le droit d'accès aux soins pour les résidents dans le besoin est le principe essentiel qui devrait régir le développement du secteur privé d'hébergement. Ils tiennent d'ailleurs à préciser que leur préoccupation à l'égard de la qualité des soins et des services vise aussi la situation actuelle qui prévaut dans le secteur public d'hébergement.

Il serait temps selon moi qu'on mette les sous et la volonté indispensable pour offrir à nos vieillards (et bientôt à nous) des services de qualité et suffisants. Pour ce faire nous devons impliquer tous les partenaires, qu'ils soient privés ou non. C'est la meilleure façon d'éviter les abus qui ne sont pas toujours causés par l'appât du gain mais par ignorance et manque cruel de ressource.

(gestionnaire du réseau public)

Les propriétaires rencontrés se sont aussi longuement entretenus sur l'enjeu de l'accès aux services, mettant surtout en cause la collaboration du réseau public. Selon eux, les résidences privées doivent être des milieux ouverts qui ne gardent pas les locataires en vase clos. Ils se considèrent honnêtes dans l'identification des services qu'ils peuvent et ne peuvent pas offrir et développent des habiletés pour aller chercher les ressources et les services externes. La collaboration du réseau public de la santé s'avère toutefois longue à acquérir et loin d'être évidente. Les obstacles identifiés sont nombreux: les ressources publiques disponibles pour les résidents en perte d'autonomie varient d'un territoire à l'autre, sont inégales et les critères d'accès restent imprécis (que ce soit pour le soutien à domicile offert par les CLSC, pour l'évaluation en courte durée gériatrique, etc.). Ce commentaire écrit va dans le même sens:

\section{Vieux réfugié, Palestine}

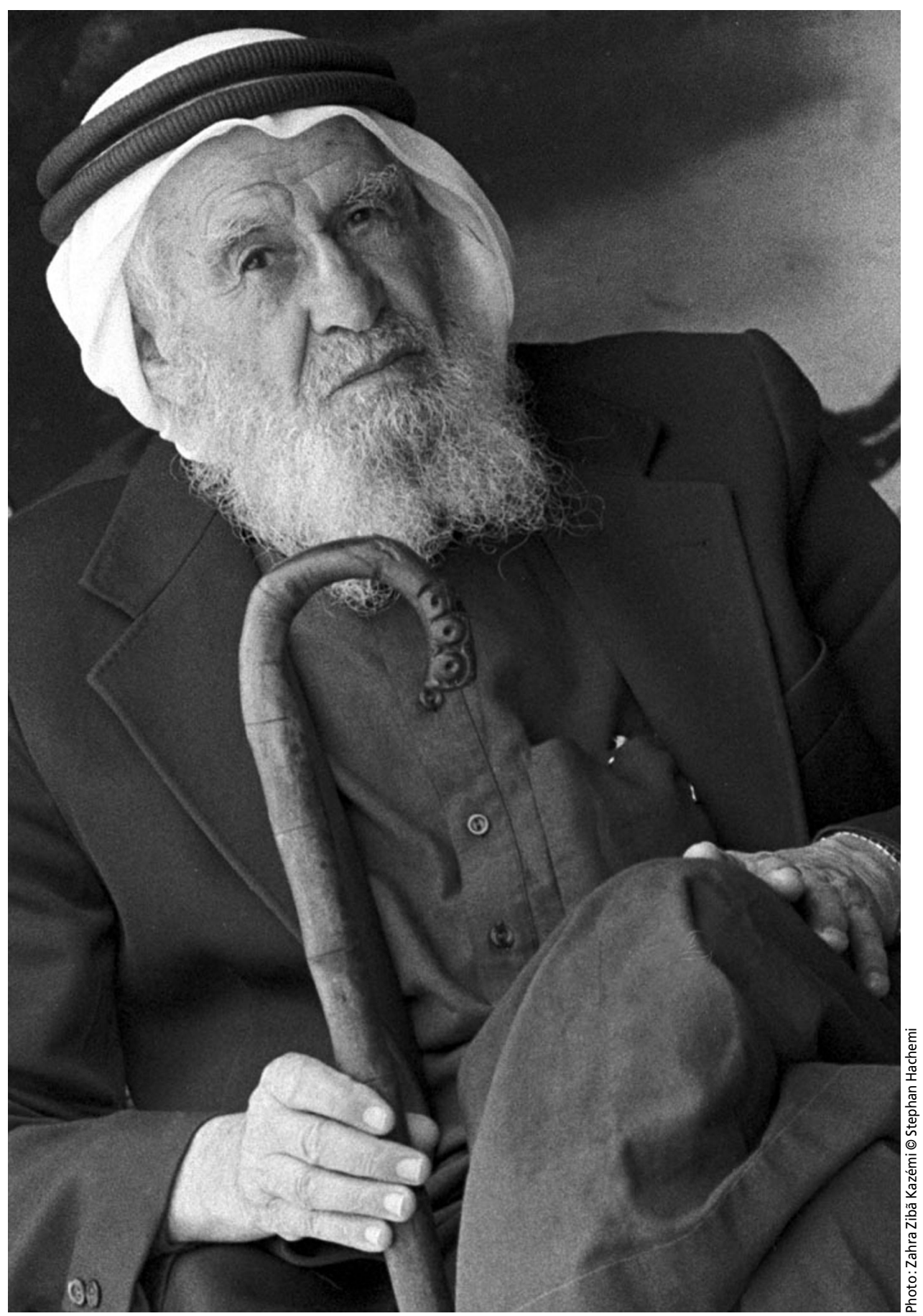


Que le CLSC respecte que l'appartement du résident qui vit chez nous soit considéré comme son domicile et qu'il a le devoir de venir donner les soins à domicile, tels les bains, les pansements et autres. J'aurais beaucoup de commentaires au sujet de CLSC, mais pas assez de lignes disponibles. (propriétaire)

Les propriétaires s'entendent pour reconnaître que lorsque c'est le réseau public qui les sollicite pour placer quelqu'un et «vider un lit», ça va très vite: "dans la journée même parfois». Le temps d'attente est tout autre lorsque ce sont eux qui demandent une collaboration au réseau public pour relocaliser un cas difficile (qui a des comportements perturbateurs par exemple) ou pour évaluer un résident et réajuster sa médication.

Ainsi, malgré des principes généreux d'universalité, d'accessibilité et de gratuité des soins et services de santé, leur exercice est soumis à de multiples réserves et conditions qui en minimisent grandement la portée (Molinari, 1996; Lajoie, 1994). Le resserrement des critères d'admission en institution publique (CHSLD) et d'allocation des services de soutien à domicile (CLSC), auxquels les résidents en perte d'autonomie auraient théoriquement droit, n'en sont que quelques exemples. Le Vérificateur général du Québec, dans son rapport à l'Assemblée nationale pour l'année 2001-2002, consacre un chapitre entier pour illustrer et dénoncer le manque de services d'hébergement offerts aux personnes âgées en perte d'autonomie. Ses travaux démontrent clairement que les CLSC ne sont pas en mesure d'offrir les services à domicile aux résidents en perte d'autonomie, même à ceux en attente d'hébergement en CHSLD.

\section{LA PROTECTION CONTRE LES ABUS}

Depuis une dizaine d'années, la problématique de la maltraitance occupe une place importante dans la recherche et la documentation scientifique en gérontologie (Spencer, 1994; CDPDJ, 2001). Les résultats de la présente étude traduisent une inquiétude face à la prévalence des abus en résidences privées mais n'adoptent pas un ton alarmiste.

La discussion entourant la problématique des abus et mauvais traitements à l'endroit des résidents âgés a été très animée dans le groupe réunissant les intervenants du réseau public, lesquels reconnaissent l'existence de pratiques abusives dans les grandes institutions CHSLD: surutilisation des contentions, nombre de bains et changements de couches insuffisants, etc. Quant aux mauvais traitements en résidence privée, ils s'entendent, sur la base de leurs expériences, pour dire que les principaux

IL S'AVÈRE IMPÉRIEUX DE TENIR COMPTE,

DANS LA DÉFINITION ET L'ÉLABORATION

DES FUTURES POLITIQUES SOCIALES,

DE LA DIVERSITÉ DES RESSOURCES MARCHANDES,

DE MÊME QUE DES INÉGALITÉS SOCIALES QU'ELLES REFLÈTENT,

ENTRETIENNENT ET PERPÉTUENT.

facteurs de risque sont l'isolement des résidences, l'épuisement des responsables et les difficultés financières. Les petites résidences, caractérisées par l'insuffisance de personnel pour partager les tâches et la pression, présentent «un haut niveau de risque d'être excédées et de développer des comportements négligents ou abusifs». Les gens s'inquiètent; les ressources privées, surtout les petites, sont de plus en plus débordées, ont peu de répit et présentent des problèmes de rentabilité. De nombreux témoignages d'épuisement ont été recueillis chez les propriétaires. À titre d'exemple:

Les directeurs et directrices en résidences privées sont épuisés. Nous avons besoin d'un soutien. Merci beaucoup de vous préoccuper de ce qui se passe en résidence privée et surtout de nous demander notre opinion.

Peu de ressources - peu d'aide beaucoup de travail nous allons vers l'épuisement des ressources. Le gouvernement travaille contre nous. J'en aurais tellement long à dire.

\section{DISCUSSION}

PLURALISME ET INÉGALITÉS SOCIALES

Les travaux que nous avons menés à ce jour sur les résidences privées pour personnes âgées et les résultats de la présente étude mettent en évidence l'important écart de perception qui sépare les secteurs privé et public. Nous sommes en présence de deux visions, voire de deux cultures différentes. D'un côté, les gestionnaires et intervenants du réseau de la santé et des services sociaux apparaissent inquiets des conditions de vie en résidences privées, ce qui se traduit par l'attribution de scores significativement plus bas que ceux octroyés par les propriétaires pour le respect des droits des résidents (cf. tableau 1). Il faut reconnaître que les intervenants du secteur public agissent presque uniquement en «bout de ligne», lorsque la situation s'est dégradée. Ce contexte d'intervention, conjugué à une certaine morosité qui sévit dans le réseau, teinte inévitablement leur lecture. Il contribue à comprendre cette culture, dite «de la problématique», qui s'appuie sur un diagnostic sévère et interpelle leur désir d'intervenir. La documentation en gérontologie a fait largement état du scepticisme des acteurs du réseau public, surtout les intervenants, face aux services non professionnels (Neysmith, 1999; Lesemann et Martin, 1993). D'ailleurs, unanimement ou presque (97\%), les artisans du public se sont prononcés en faveur d'une intervention étatique, revendiquant plus de normes et de standards.

De leur côté, les propriétaires de résidences privées fournissent une appréciation très positive de la qualité des services qu'ils dispensent. Cette auto-évaluation favorable va certes dans le sens de leur intérêt; il s'agit de leur gagne-pain, mais elle traduit aussi leur fort sentiment de fierté face à leur réalisation et leur spécificité, comme en témoignent les commentaires des propriétaires en ce qui a trait à la dimension humaine et chaleureuse de leur résidence. Cette perception positive de leur contribution ne les empêche pas toutefois d'être plutôt favorables à l'intervention étatique et de revendiquer de l'aide. Rappelons les nombreux témoignages faisant état d'essoufflement et de risque d'épuisement.

Cette diversité des points de vue et la contradiction apparente des résultats reflètent à notre avis les importantes disparités et inégalités entre les résidences privées et entre leurs résidents. Il se développe, à l'intérieur même du secteur privé, un système à deux vitesses. Il appert que la dynamique prix-compétition engendre deux standards de résidence: un pour les non-pauvres et un autre, inférieur, pour les pauvres (Soderstrom, 1987). Si quelques résidences luxueuses, dotées de multiples services spécialisés, ciblent les retraités nantis, la majorité (surtout celles de petite ou moyenne taille) sont confrontées aux limites financières de leur clientèle type, soit des femmes seules, âgées de plus de 75 ans. Or, dans ces petites ressources, les difficultés économiques risquent d'inciter les propriétaires à garder des résidents devenus très lourds, par crainte de se retrouver avec un lit inoccupé. À l'opposé, il y a lieu de s'inquiéter aussi du comportement parfois abusif des propriétaires qui, 
profitant d'une forte demande et de listes d'attente imposante, cherchent à évincer les résidents nécessitant une surveillance et des soins afin d'accueillir des personnes plus autonomes. La présence accrue de personnes âgées en perte d'autonomie, particulièrement celles à faible revenu, soulève de façon particulière la question de leur droit à des conditions décentes de vie et à l'accès aux soins et aux services requis par leur condition. Ces considérations renvoient à une réflexion sociale et éthique qui met en jeu les principes d'équité et de justice sociale. Est-il possible de faire émerger de ces résultats et ce, malgré les logiques différentes qui les animent, un projet qui pourrait servir de guide dans l'élaboration des politiques sociales?

Il s'avère impérieux de tenir compte, dans la définition et l'élaboration des futures politiques sociales, de la diversité des ressources marchandes, de même que des inégalités sociales qu'elles reflètent, entretiennent et perpétuent. Nous ne saurions trop insister sur les pièges de l'approche normative qui consisterait à imposer un ensemble de normes ou de standards «mur à mur» sans égard aux conditions d'existence dans ces milieux. L'hétérogénéité des résidences est une caractéristique à préserver pour répondre aux besoins diversifiés des aînés (Maltais, 1999). Par surcroît, trop d'exigences ne pourront être respectées par les petites résidences, risquant d'exacerber les tensions déjà pressenties et de compromettre à moyen terme leur survie. Dans ce sens, la recherche d'une plus grande équité et la lutte contre les inégalités dénoncées devraient constituer l'objectif premier des futures politiques. Il s'avère impérieux de reconnaître la contribution du privé dans l'hébergement des personnes âgées vulnérables et de développer des modalités de soutien en termes d'assistance financière et de services directs par les organismes du secteur public. Il y a ici tout un défi qui s'ouvre en termes de renouvellement des pratiques sociales et des rapports privépublic.

\section{Bibliographie}

AUBRY, F. (2004). "Les résidences privées à but lucratif pour personnes âgées ", dans M. CHARPENTIER et Y. VAILLANCOURT (dir.), Cahiers du LAREPPS, Laboratoire de recherche sur les pratiques et politiques sociales, École de travail social, UQAM (à paraître).

BOITTE, P. (1995). Éthique, justice et santé. Allocation des ressources en soins dans une population vieillissante, Montréal, ArtelFides.

BOISVERT, G. (1997). «L'éthique comme suppléance politique: une approche postmoderniste », dans G. GIROUX (dir.), La pratique sociale de l'éthique, Québec, Bellarmin.
BRAVO, G., M. CHARPENTIER, M.F. DUBOIS, P. DeWALS et A. ÉMOND (1998). «Profile of residents in unlicensed homes for the aged in the Eastern Townships of Quebec», Canadian Medical Association Journal, no 159 , p. 143-148.

BRISSETTE, L (1992). "Le développement du secteur privé de l'hébergement pour personnes âgées au Québec; entre un processus concret et un "silence permissif" ", Revue canadienne de politique sociale, $\mathrm{n}^{\circ} 29-30$, p. 158-167.

CHARPENTIER, M. (2002). Priver ou privatiser la vieillesse? Entre le domicile à tout prix et le placement à aucun prix, Sainte-Foy, Presses de l'Université du Québec, 206 p.

CHARPENTIER, M., N. DELLI-COLLI et L. DALPÉ (2000). «L'orientation des personnes âgées en perte d'autonomie dans un contexte de rareté des ressources publiques d'hébergement», Intervention, $\mathrm{n}^{\circ} 112$, p. 70-78.

COMMISSION DES DROITS DE LA PERSONNE DU QUÉBEC ET DES DROITS DE LA JEUNESSE (2001). L'exploitation des personnes âgées: vers un filet de protection resserré. Rapport de consultation.

CONSEIL DE LA SANTÉ ET DU BIENÊTRE (1997). Évolution des rapports publicprivé dans les services de santé et services sociaux. Rapport remis au ministre de la Santé et des Services sociaux, Gouvernement du Québec.

CONSEIL DES AÎNÉS (2000). Avis sur l'hébergement en milieux de vie substituts pour les aînés en perte d'autonomie. Par D. GAGNON et A. MICHAUD, Gouvernement du Québec.

DELPÉRÉE, N. (1991). La protection des droits et libertés des citoyens âgés. Thèse de doctorat en droit, prix Jean-Toigne, Toulouse, Université des sciences sociales.

GOUVERNEMENT DU QUÉBEC (juin 2004). Registre des résidences privées pour personnes âgées avec services.

KENDALL, J. (2001). "Grande-Bretagne: une économie plurielle de soins bouleversés par les "quasi-marchés" ", dans J.-L. LAVILLE et M. NYSSENS (dir.), Les services sociaux entre associations, Etat et marché: l'aide aux personnes âgées, Paris, La Découverte, p. 91-117.

LAJOIE, A. (1994). «Le droit aux services: une réforme en peau de chagrin", dans V. LEMIEUX et al. (dir.), Le système de santé au Québec: organisations, acteurs et enjeux, Cowansville, Yvon Blais, p.129-

LESEMANN, F. et C. MARTIN (1993). «Solidarités familiales et politiques sociales », dans F. LESEMANN et C. MARTIN (dir.), Les personnes âgées: dépendance, soins et solidarités familiales: comparaisons internationales, Paris, La Documentation française.

LOI MODIFIANT LA LOI SUR LES SERVICES DE SANTÉ ET LES SERVICES SOCIAUX CONCERNANT LES RÉSIDENCES POUR PERSONNES ÂGÉES, 2002, c. 36, art. 1 .
MALTAIS, D. (1999). "Vivre en résidence pour aînés: le format est-il la formule? ", Santé mentale au Québec, vol. XXIV, $\mathrm{n}^{\circ} 1$, p. $173-198$.

MINISTÈRE DE LA SANTÉ ET DES SERVICES SOCIAUX (2003). Un milieu de vie de qualité pour les personnes hébergés en CHSLD, orientations ministérielles.

NEYSMITH, S. (dir.) (1999). Critical issues for future social work practice with aging persons, New York, Columbia University Press.

PAILLÉ, P. (1996). «De l'analyse qualitative en général et de l'analyse thématique en particulier", Revue de l'Association pour la recherche qualitative, vol. 15, p. 179-195.

SPENCER. C. (1994). Les mauvais traitements et la négligence envers les personnes âgées en milieu institutionnel, Ottawa, Santé Canada.

SODERSTROM, L. (1987). Privatisation: adopt or adapt? Commission d'enquête sur les services de santé et services sociaux, Synthèse critique n ${ }^{\circ} 36$, Québec.

VAILLANCOURT, Y. (1997). Vers un nouveau partage des responsabilités dans les services sociaux et de santé: rôles de l'État, du marché, de l'économie sociale et du secteur informel. Rapport de recherche, Laboratoire de recherche sur les pratiques et les politiques sociales (LAREPPS), Université du Québec à Montréal.

VAILLANCOURT, Y., D. BOURQUE, F. DAVID et E. OUELLET (1987). La privatisation des services de santé et services sociaux. Commission d'enquête sur les services de santé et services sociaux, Annexe $\mathrm{n}^{\circ}$ 36, Les Publications du Québec.

VÉRIFICATEUR GÉNÉRAL DU QUÉBEC (2002). Rapport à l'assemblée nationale pour l'année 2001-2002. Tome 1, chap. 2: Services d'hébergement offerts aux personnes âgées, Québec.

VÉZINA, A., D. PELLETIER et J. ROY (1994). Les résidences privées et les HLM pour personnes âgées de la région de Québec: profils des ressources et clientèles et paramètres d'un cadre de références. Centre de recherche sur les services communautaires, Faculté des sciences sociales, Université Laval.

\section{Note}

1. Soulignons que nous menons présentement une recherche qui, en partenariat avec le Secrétariat aux aînés et la Table de concertation des aînés de Montréal, vient donner la parole aux résidents âgés. Intitulée "Droits et empowerment des personnes âgées vivant en milieu d'hébergement», l'étude vise à recueillir le point de vue des résidents sur leurs conditions de vie et sur le respect de leurs droits fondamentaux. 